\section{(6) OPEN ACCESS}

\title{
Incidence and relative risk of hearing disorders in professional musicians
}

\author{
Tania Schink, ${ }^{1}$ Gunter Kreutz, ${ }_{1}^{2}$ Veronika Busch, ${ }^{3}$ Iris Pigeot, ${ }^{4,5}$ Wolfgang Ahrens ${ }^{5,6}$
}

For numbered affiliations see end of article.

\section{Correspondence to} Dr Wolfgang Ahrens, Leibniz Institute for Prevention Research and EpidemiologyBIPS, Department of Epidemiologic Methods and Etiologic Research Achterstraße 30, Bremen 28359, Germany; ahrens@bips.uni-bremen.de

Received 16 February 2014 Accepted 4 March 2014 Published Online First 30 April 2014

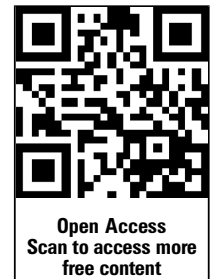

CrossMark

To cite: Schink T, Kreutz $G$, Busch V, et al. Occup Environ Med 2014:71: 472-476.

\begin{abstract}
Background Hearing disorders have been associated with occupational exposure to music. Musicians may benefit from non-amplified and low-intensity music, but may also have high risks of music-induced hearing loss. Aims To compare the incidence of hearing loss (HL) and its subentities in professional musicians with that in the general population.

Methods We performed a historical cohort study among insurants between 19 and 66 years who were employed subject to social insurance contributions. The study was conducted with data from three German statutory health insurance providers covering the years 2004-2008 with about 7 million insurants. Incidence rates with $95 \% \mathrm{Cls}$ of $\mathrm{HL}$ and the subentities noiseinduced hearing loss (NIHL), conductive $\mathrm{HL}$, sensorineural $\mathrm{HL}$, conductive and sensorineural $\mathrm{HL}$, as well as tinnitus were estimated stratified by age, sex and federal state. A Cox regression analysis was conducted to estimate adjusted HRs and two-sided $95 \% \mathrm{Cls}$ for $\mathrm{HL}$ and its subentities.
\end{abstract}

Results More than 3 million insurants were eligible, of whom 2227 were identified as professional musicians $(0.07 \%)$. During the 4 -year observation period, 283697 cases of $\mathrm{HL}$ were seen, 238 of them among professional musicians $(0.08 \%)$, leading to an unadjusted incidence rate ratio of 1.27 . The adjusted hazard ratio of musicians was $1.45(95 \% \mathrm{Cl} 1.28$ to 1.65) for $\mathrm{HL}$ and $3.61(95 \% \mathrm{Cl} 1.81$ to 7.20$)$ for NIHL.

Conclusions Professional musicians have a high risk of contracting hearing disorders. Use of already available prevention measures should reduce the incidence of $\mathrm{HL}$ in professional musicians.

\section{INTRODUCTION \\ Background}

Over the past 50 years, medical problems in professional musicians have been studied, with some emphasis on hearing. ${ }^{1-7}$ In particular, there have been several attempts to assess the risks of hearing loss (HL) in response to exposure to music, mainly in classical musicians ${ }^{8-12}$ and in rock/pop musicians. ${ }^{13-15}$ Noise-induced hearing loss (NIHL), plausibly interpreted as music-induced hearing loss when musicians are investigated, was seen in up to $58 \%$ of classical, and up to $49 \%$ of rock/pop, musicians. ${ }^{16}$ It should be noted that findings are based primarily on subclinical measures such as pure tone audiometry and in fewer cases, on reports on hearing problems such as hyperacusis, loss of hearing sensitivity and tinnitus (cf. Tables 1 and 2 in Zhao et al 2010 ${ }^{16}$ ). Therefore, the clinical implications, particularly of

\section{What this paper adds}

- Professional musicians have an increased risk of hearing-related disorders, known to be associated with occupational exposure to high sound pressure levels.

- A large claims database was used to identify professional musicians and assess their risks of hearing-related disorders in comparison with the general population.

- A nearly fourfold higher adjusted HR for noise-induced hearing loss and a 57\% higher adjusted HR for tinnitus was found for professional musicians in comparison with the general population.

- The new evidence underpins the need to implement strategies for hearing protection in professional musicians.

observed permanent or temporary shifts of hearing thresholds, have remained unclear.

NIHL seems to be provoked by both extrinsic and intrinsic factors. ${ }^{16}{ }^{17}$ For example, extrinsic factors include the number of years of exposure to high levels of sound and the position of the players in the orchestra on stage, in an orchestra pit, or near loud speakers, ${ }^{17}$ as well as the instruments being played. ${ }^{18}$ By contrast, more intrinsic factors related to individual differences in psychophysiological proneness to hearing disorders among musicians appear less well understood. Not surprisingly, previous studies on the size of the risk of hearing problems in professional musicians have been inconclusive. ${ }^{16}$ Moreover, the extent of damage and details of medical treatment are often not documented. ${ }^{17}$

Some studies have argued against a significant relationship between music exposure and hearing problems, with reservations based on methodological concerns. For example, Karlsson, Lundquist and Olaussen suggested that the sound exposure criteria for industrial noise are not valid when the relevant sound sources are acoustic instruments of a symphonic orchestra. ${ }^{19}$ Further problems arise from the fact that definitions of NIHL may vary from study to study, leaving open whether or not clinical implications can be drawn; also, some studies depend upon identifying a well-matched reference population. ${ }^{20}{ }^{21}$ These concerns notwithstanding, Zhao $e a^{16}$ suggested that there could be 
Table 1 Characteristics of insurants eligible for the study cohort

\begin{tabular}{|c|c|c|}
\hline Variable & Musicians $(\mathrm{N}=2227)$ & Others ( $N=3340718$ ) \\
\hline \multicolumn{3}{|l|}{ Sex } \\
\hline Male & $1257(56.4)$ & $1903151(57.0)$ \\
\hline Female & $970(43.6)$ & $1437567(43.0)$ \\
\hline \multicolumn{3}{|l|}{ Age at cohort entry (years) } \\
\hline Mean (SD) & $39.7(11.56)$ & $39.7(11.56)$ \\
\hline Median (quartiles) & $40.0(30.0 ; 48.0)$ & $40.0(30.0 ; 48.0)$ \\
\hline \multicolumn{3}{|l|}{ Population density } \\
\hline City & $1311(58.9)$ & $1276648(38.2)$ \\
\hline Suburb & $658(29.5)$ & $1445336(43.3)$ \\
\hline Rural surrounding area & $115(5.2)$ & 352331 (10.5) \\
\hline Rural & $135(6.1)$ & $250684(7.5)$ \\
\hline Other/unknown & $8(0.4)$ & $15719(0.5)$ \\
\hline
\end{tabular}

little doubt about music-induced hearing loss as a potential problem of professional musicianship.

In summary, assessments of professional musicians' risks of permanent damage of inner ear structures are compromised by a lack of comparable population data, and population-based studies are needed to assess musicians' proneness to hearing problems.

Here we present a large-scale epidemiological study to assess the risk of musicians contracting hearing-related disorders on the basis of insurance claims related to hospital admission and outpatient diagnoses. Specifically, we ask to what extent professional musicians have a higher incidence of hearing problems in comparison with the general population.

\section{Objective}

The objective of this study was to compare the incidence of HL and its subentities in professional musicians with that in the general population.

\section{METHODS}

\section{Data source}

The study was conducted with data from three German statutory health insurance providers (SHIs) covering the years
2004-2008 with about 7 million insurants. The database contains demographic information about each insurant as well as information on hospital admissions, outpatient visits to a doctor and outpatient prescriptions. ${ }^{22}$ Since outpatient visits to a doctor are reimbursed quarterly during the year, an algorithm was developed to allocate an exact date for outpatient diagnoses. All diagnoses, outpatient as well as inpatient, were coded according to the German modification of the International Classification of Diseases (ICD-10-GM). Analyses of the age and sex distribution, number of hospital admissions and drug use have shown the database to be representative for Germany. $^{23} 24$ The use of health insurance data for scientific research is regulated by the Code of Social Law (SGB X). Informed consent was not required by law, since the study was based on pseudonymous data.

\section{Study design}

We performed a historical cohort study among insurants aged between 18 and 66 years who were employed subject to social insurance contributions and who were continuously insured for 12 months before cohort entry. Cohort entry was defined as the first date after 1 January 2005 on which the insurant had been continuously insured for more than 365 days, had his/her 19th to 66th birthday in the year of cohort entry and had had no diagnosis of hearing loss during the preceding 12 months. Cohort exit was defined as the first of the following dates: 31 December of the year in which the insurant had his/her 66th birthday, end of study period — that is, 31 December 2008, day of first diagnosis of HL and interruption or end of insurance, which was defined as the end date of the first insurance period after cohort entry. Re-entry after cohort exit was not possible.

\section{Definition of professional musician}

Registration with a given SHI is usually done through the employer who records key sociodemographic characteristics of the insurant, including his/her occupation. Freelance musicians are eligible to register themselves via a specific artists' social insurance agency (Künstlersozialkasse). Professional musicians were identified using the Standard Classification of Occupations, which is used by the Federal Employment Agency. ${ }^{25}$ The corresponding code comprises rock/pop or classical instrumental musicians, singers, conductors and composers. A professional musician was defined as an insurant who had had

Table 2 Person-time, number of cases and incidence of hearing loss and its subentities

\begin{tabular}{|c|c|c|c|c|c|}
\hline Disease group & $\begin{array}{l}\text { Occupational } \\
\text { group }\end{array}$ & $\begin{array}{l}\text { Person-time (in } 100000 \\
\text { person-years) }\end{array}$ & $\begin{array}{l}\text { Cases } \\
\text { (N) }\end{array}$ & $\begin{array}{l}\text { Incidence (per } 100000 \\
\text { person-years) }\end{array}$ & IRR (95\% CI) \\
\hline \multirow[t]{2}{*}{ Hearing loss } & Musicians & 0.07 & 238 & 3621 & 1.27 (1.14 to 1.39$)$ \\
\hline & Others & 99.11 & 283459 & 2860 & 1 (Ref.) \\
\hline \multirow[t]{2}{*}{ Noise-induced hearing loss } & Musicians & 0.07 & 8 & 122 & $3.51(2.82$ to 4.21$)$ \\
\hline & Others & 99.11 & 3433 & 35 & 1 (Ref.) \\
\hline \multirow[t]{2}{*}{ Conductive hearing loss } & Musicians & 0.07 & 12 & 183 & $1.12(0.55 ; 1.68)$ \\
\hline & Others & 99.11 & 16193 & 163 & 1 (Ref.) \\
\hline \multirow[t]{2}{*}{ Sensorineural hearing loss } & Musicians & 0.07 & 42 & 639 & 0.91 (0.60 to 1.21$)$ \\
\hline & Others & 99.11 & 69945 & 706 & 1 (Ref.) \\
\hline \multirow{2}{*}{$\begin{array}{l}\text { Combined conductive and sensorineural hearing } \\
\text { loss }\end{array}$} & Musicians & 0.07 & 2 & 30 & 0.51 (0.30 to 1.71$)$ \\
\hline & Others & 99.11 & 5944 & 60 & 1 (Ref.) \\
\hline \multirow[t]{2}{*}{ Tinnitus } & Musicians & 0.07 & 148 & 2252 & $1.45(1.29$ to 1.61$)$ \\
\hline & Others & 99.11 & 153737 & 1551 & 1 (Ref.) \\
\hline
\end{tabular}


at least one insurance period in the study period from 1 January 2004 through 31 December 2008 coded as 'musician'.

\section{Case definition}

Cases were defined as cohort members with a diagnosis of HL - that is, an outpatient or hospital diagnosis with at least one of the following ICD-10-GM codes: H83.3 noise effects on inner ear, H90 conductive and sensorineural hearing loss or H93.1 tinnitus. Additionally, we defined four subentities of HL: NIHL if the ICD-10-GM code was H83.3, conductive HL if the codes H90.0 to H90.2 were used, sensorineural HL if the codes H90.3 to H90.5 were used, combined conductive and sensorineural HL if the code H90.6 was used and tinnitus if the code H93.1 was used.

\section{Statistical analysis}

We calculated incidence rates of HL, and incidence rates for the subentities NIHL, conductive HL, sensorineural HL, combined conductive and sensorineural HL and tinnitus stratified by age, sex and federal state. CIs for the incidence rates were estimated by the substitution method assuming a Poisson distribution for the number of events. ${ }^{26}$

A Cox proportional hazards model was calculated to estimate adjusted HRs and two-sided 95\% CIs for HL and its subentities, taking into account the differing observation periods between cohort members. The time scale for the Cox regression was the number of days each person was observed in cohort.

In the sensitivity analyses, adjustments were made for sex and age at cohort entry (continuous variables), for population density of place of residence (categorised as cities, concentrated surrounding areas, rural surrounding areas and rural areas), SHI and federal state.

All statistical analyses were done using SAS V.9.2 (SAS Institute Inc, Cary, North Carolina, USA) and the proportional hazards assumption was tested with the ASSESS option.

\section{RESULTS}

More than 3 million insurants with employment subject to social insurance contributions were included in the cohort, of whom 2227 insurants could be identified as professional musicians $(0.07 \%)$. Table 1 summarises demographic and regional distribution information of professional musicians in comparison with all other professions. Age and sex distribution were

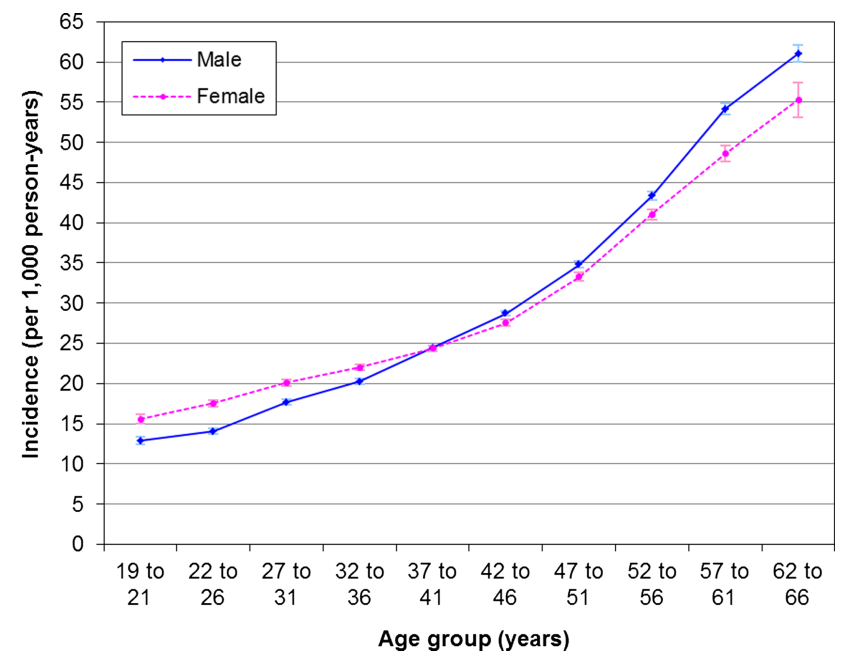

Figure 1 Incidence rate of hearing loss by sex and age. similar; however, musicians were over-represented in Berlin (12.9\% of the musician subcohort compared with $6.7 \%$ of the reference cohort). A higher percentage of musicians lived in cities (58.9\% vs $38.2 \%$ ) and only $11.2 \%$ lived in rural or rural surrounding areas compared with $18.1 \%$ of the reference cohort representing the general population.

During the study period, 283697 cases of HL were newly diagnosed, 238 of them among professional musicians (0.08\%), leading to an unadjusted incidence rate ratio of 1.27 (table 2). Musicians had a 3.51-fold higher incidence rate of NIHL and a 1.45 higher incidence rate of tinnitus than the general population. Unadjusted incidence rates for the other subentities were quite similar between musicians and the general population; all CIs overlapped. The seemingly protective effect for combined conductive and sensorineural HL was based on only two cases among musicians, leading to a broad, non-conclusive CI.

The incidence of HL was slightly higher for men (2990 vs 2678 per 100000 person-years), but was not associated with population density. As expected, incidence rates increased with age. Up to the age of 36 years the incidence of HL was higher in women, but above that age it was higher for men (figure 1). The incidence of HL did not increase monotonically with age for any of its subentities (data not shown).

Adjusting for sex and age at cohort entry by performing a Cox regression analysis yielded a HR of 1.45 (95\% CI 1.28 to 1.65) for HL in musicians (table 3 ). Men were at slightly higher risk of HL with a HR of 1.01 (95\% CI 1.00 to 1.02). Inclusion of additional potential confounders (population density of place of residence, SHI and federal state) as well as an interaction term for age $\times$ sex into the statistical model did not change the HRs for HL in musicians.

Among professional musicians the HR was 3.61 (95\% CI 1.81 to 7.20$)$ for NIHL and 1.57 (95\% CI 1.34 to 1.85$)$ for tinnitus (table 3 ). The HR did not differ statistically significantly from unity for the other subentities of $\mathrm{HL}$ - that is, conductive and sensorineural HL and combined conductive and sensorineural HL.

\section{DISCUSSION}

It was hypothesised that professional musicians are exposed to a significant risk of contracting hearing disorders due to high levels of sound in their working environment. We found an almost fourfold higher adjusted HR for NIHL and a $57 \%$

Table 3 HR estimates and corresponding 95\% Cls for hearing loss, noise-induced hearing loss and tinnitus

\begin{tabular}{lll}
\hline & HR & 95\% Wald CI \\
\hline $\begin{array}{l}\text { Hearing loss } \\
\text { Musician }\end{array}$ & 1.45 & \\
$\quad$ Male sex & 1.01 & $(1.276$ to 1.646$)$ \\
$\quad$ Age at cohort entry & 1.04 & $(1.002$ to 1.017$)$ \\
Noise-induced hearing loss & & $(1.035$ to 1.036$)$ \\
$\quad$ Musician & 3.61 & \\
$\quad$ Male sex & 2.10 & $(1.806$ to 7.201$)$ \\
$\quad$ Age at cohort entry & 1.001 & $(1.947$ to 2.273$)$ \\
Tinnitus & & $(0.998$ to 1.004$)$ \\
$\quad$ Musician & 1.57 & $(1.337$ to 1.845$)$ \\
$\quad$ Male sex & 0.99 & $(0.922$ to 0942$)$ \\
Age at cohort entry & 1.02 & $(1.021$ to 1.022$)$ \\
\hline
\end{tabular}


higher adjusted HR for tinnitus for professional musicians in comparison with the general population. Case numbers were too small for conclusive results for the other subentities of HL - that is, conductive and sensorineural HL and combined conductive and sensorineural HL.

Zhao et $a l^{16}$ proposed that exposure to loud music might cause hearing symptoms, including temporary threshold shifts and tinnitus. The review by these authors, however, is primarily based on field studies using pure tone audiometry for the assessment of temporary and permanent hearing threshold shifts. Some reports have also described hearing problems such as hyperacusis or loss of hearing sensitivity, but interpretations were compromised owing to small population sizes and, in many cases, the absence of adequate reference groups.

To our knowledge this is the largest study comparing the risk of HL among professional musicians with that in the general population. The strengths of our study are the size of the database and its representativeness. There is no potential for selection bias due to selective non-response of potential study participants. All information was recorded prospectively so that recall bias was avoided.

Karlsson et $a l^{19}$ have pointed out that industrial noise may not be comparable with loud music. While chronic exposure to industrial noise has been clearly linked with hearing damage, including loss of hearing sensitivity, research on professional musicianship suggests increases of hearing sensitivity rather than decreases. For example, musicians have been shown to develop enhanced sensitivity to meaning in non-musical sound, ${ }^{27}$ which might, to a certain extent, mask fundamental hearing deficits. Moreover, Zendel and Alain ${ }^{28}$ found that musicians might receive benefit from their training such that age-related hearing loss is attenuated. However, these observations may not apply to specific subgroups. In light of our results, it might be that many musicians prone to $\mathrm{HL}$ do contract NIHL before age-related HL emerges. Our data suggest that in professional musicians the risks of music-induced HL outweigh by far the potential benefits for hearing ability as reported by Zendel and Alain. ${ }^{28}$ Unfortunately, our data do not allow a distinction between different types of musicians (eg, those using acoustic vs electronic instruments).

Given the significant long-term risks to professional musicians of contracting hearing disorders, their management in educational settings seems appropriate. ${ }^{29}$ This might entail, for example, increasing conductors' awareness of loud music while rehearsing and during concerts with large orchestras. Furthermore, musicians should be offered protection by in-ear devices and installation of sound-protecting shields between the sections of an orchestra. Some of these measures, such as in-ear sound protection, should also apply to professional musicians playing in rock bands, or when electric sound amplifiers are used. Some of these efforts may significantly reduce this risk for future generations. However, without internationally accepted policies to deal with the problem it is likely that musicians will continue to have a risk of contracting hearing disorders.

\section{Strengths and limitations}

Owing to the nature of secondary data, not all variables can be assessed in the desired detail. In particular, no information on the type of instrument, the kind of music, or the setting in which the musician worked is included in our claims database. The group of musicians defined by the occupational code is quite diverse, including instrumental musicians as well as singers, conductors and composers. Since certain subgroups of musicians are more exposed to higher sound levels than others, we assume that this diversity will dilute the effects, leading to an underestimation of the risk.

There is also a potential for non-differential misclassification of the outcome. On the one hand, some diagnoses of HL might be incorrect; on the other, some cases of HL might not be diagnosed at all and therefore be missed in our study. It is unlikely that the observed large effects are fully explained by nondifferential misclassification.

However, there is potential for detection bias. On the one hand, musicians might be reluctant to acknowledge hearing problems, because of fear of stigmatisation. For example, perfectionism (worry about the judgement of others) has been identified as one source of stress in professional orchestras. ${ }^{30}$ On the other hand, it is possible, that fewer cases of HL are missed in professional musicians, who might be more sensitive to hearing problems than the general population. However, musicians do not show marked differences in health-related measures as compared with the general population. ${ }^{31}$ Williamson and Thompson ${ }^{32}$ suggested that although conservatoire students already experience health problems that are related to their musical performance and practice behaviours, they tend to seek medical advice from their teachers before turning to the general health system. Further studies suggest that there is a clear need for specialist interventions in health problems of professional musicians. ${ }^{33}$ In health systems that are not well-prepared to deal with music-related disorders and health issues, it seems unlikely that professional musicians show strong inclinations to seek health advice for problems including those of hearing and auditory dysfunction.

NIHL may be caused by one-time acoustic trauma due to explosions, gunfire or firecrackers. NIHL may, however, also develop gradually by repeated exposure to loud noise. Risk factors for non-congenital sensorineural HL and conductive HL are age, chronic noise exposure, genetic factors and infections such as otitis media. Besides infections other diseases such as physical injury, Eustachian ear dysfunctions and otosclerosis may lead to conductive hearing loss. In addition, it is well known that some drugs (eg, aminoglycosides) are ototoxic and can lead to irreversible cochlear and vestibular dysfunction. However, none of these risk factors occurs sufficiently frequently to be a strong confounder. Therefore, in the main analysis we adjusted for age and sex only and additionally, in the sensitivity analyses, for federal state, population density of place of residence (to cover regional differences) and SHI (as a proxy for socioeconomic status). Risk estimates remained virtually unchanged after these adjustments.

Given the number of professional musicians and the severity of the outcome, leading to occupational disability and severe loss of quality of life, hearing loss in professional musicians is of high public health importance. ${ }^{34}$ Our data provide evidence of the need for prevention measures.

\footnotetext{
Author affiliations

${ }^{1}$ Department of Clinical Epidemiology, Leibniz Institute for Prevention Research and Epidemiology-BIPS, Bremen, Germany

${ }^{2}$ Department of Music, School of Linguistics and Cultural Studies, Carl von Ossietzky Universität Oldenburg, Oldenburg, Germany

${ }^{3}$ Department of Musicology and Music Education, Faculty of Cultural Studies (FB 9), University of Bremen, Bremen, Germany

${ }^{4}$ Department of Biometry and Data Management, Leibniz Institute for Prevention

Research and Epidemiology-BIPS, Bremen, Germany

${ }^{5}$ Institute of Statistics, Faculty of Mathematics and Computer Science (FB 3),

University of Bremen, Bremen, Germany

${ }^{6}$ Department of Epidemiologic Methods and Etiologic Research, Leibniz Institute for

Prevention Research and Epidemiology-BIPS, Bremen, Germany
} 
Acknowledgements The use of the data for the study was approved by the German Federal Ministry of Health. We thank all statutory health insurance providers that provided data for this study: the Allgemeine Ortskrankenkasse (AOK) Bremen/ Bremerhaven, the Techniker Krankenkasse (TK) and the hkk. We also thank Andrea Halpern for editing the manuscript.

Contributors TS implemented the study, analysed and interpreted the data and drafted and revised the paper. GK and VB contributed to the analysis, interpreted the data and drafted and revised the paper. WA designed the study. IP and WA had the initial idea for the study, contributed ideas to the analysis, interpreted the data and revised the paper. They are guarantors of the study.

Funding The study was funded in part by a grant from the Nowetas-Foundation (http://www.nowetas.de). The rest of the study was financed by BIPS's own resources.

\section{Competing interests None.}

Ethics approval Insitutional review board of the University of Bremen, Bremen, Germany.

Provenance and peer review Not commissioned; externally peer reviewed.

Open Access This is an Open Access article distributed in accordance with the Creative Commons Attribution Non Commercial (CC BY-NC 3.0) license, which permits others to distribute, remix, adapt, build upon this work non-commercially, and license their derivative works on different terms, provided the original work is properly cited and the use is non-commercial. See: http://creativecommons.org/ licenses/by-nc/3.0/

\section{REFERENCES}

1 Chesky K, Hanoch MA. Instrument-specific reports of hearing loss: differences between classical and nonclassical musicians. Med Prob Perf Art 2000;15 35-8.

2 Fearn RW. Hearing-loss in musicians. J Sound Vib 1993;163:372-8.

3 Hasson D, Theorell T, Liljeholm-Johansson $Y$, et al. Psychosocial and physiological correlates of self-reported hearing problems in male and female musicians in symphony orchestras. Int J Psychophys 2009:74:93-100.

4 Speaks C, Nelson D, Ward WD. Hearing loss in rock'n'roll musicians. J Occup Med 1970;12:216-19.

5 Teie PU. Noise-induced hearing loss and symphony orchestra musicians: risk factors, effects and management. Maryland Med J 1998;47:13-18.

6 Sataloff RT. Hearing-loss in musicians. Am J Otology 1991;12:122-7.

7 Sataloff RT. Hearing loss in singers and other musicians. Med Prob Perf Artists 1997;12:51-6.

8 Behar A, Wong W, Kunov H. Risk of hearing loss in orchestra musicians: review of the literature. Med Prob Perf Art 2006:21:164-8

9 Flach M, Aschoff E. The risk of occupational deafness in musicians. Germ Med Month 1967:12:49-54.

10 Kähäri K, Axelsson A, Hellström $P$, et al. Hearing assessment in classical orchestral musicians. Scand Audiol 2001;30:13-23.

11 Kähäri K, Axelsson A, Hellström $P$, et al. Hearing development in classical orchestra musicians: a follow-up study. Scand Audiol 2001b;30:141-9.

12 Pawlaczyk-Luszczynska M, Dudarewicz A, Zamojska M, et al. Risk assessment of hearing loss in orchestral musicians. Medycyna Pracy 2010;61:493-511.
13 Axelsson A, Lindgren F. Factors increasing the risk for hearing loss in pop musicians. Scand Audiol 1977;6:127-31

14 Axelsson A, Eliasson A, Israelsson B. Hearing in pop/rock musicians: a follow up study. Ear Hearing 1995; 16:245-53.

15 Kähäri K, Zachau G, Eklöf $M$, et al. Assessment of hearing and hearing disorders in rock/jazz musicians. Int J Audiol 2003;42:279-88.

16 Zhao F, Manchaiah VKC, French D, et al. Music exposure and hearing disorders: an overview. Int J Audiol 2010:49:54-64.

17 Palin SL. Does classical music damage the hearing of musicians? A review of the literature. Occup Med (Lond) 1994:44:130-6.

18 Hoffman JS, Cunningham DR, Lorenz DJ. Auditory thresholds and factors contributing to hearing loss in a large sample of percussionists. Med Prob Perf Art 2006;21:47-58

19 Karlsson K, Lundquist PG, Olaussen T. The hearing of symphony orchestra musicians. Scand Audiol 1983;12:257-64.

20 McBride D, Gill F, Proops D, et al. Noise and the classical musician. Br Med J 1992:305:1561-3.

21 Westmore GA, Eversden ID. Noise-induced hearing loss and orchestral musicians. Arch Otolaryngol 1981;107:761-4.

22 Pigeot I, Ahrens W. Establishment of a pharmacoepidemiological database in Germany: methodological potential, scientific value and practical limitations. Pharmacoepidemiol Drug Safety 2008;17:215-23.

23 Schink T, Garbe E. Assessment of the representativity of in-patient hospital diagnoses in the German Pharmacoepidemiological Research Database. Pharmacoepidemiol Drug Safety 2010;19:178-9.

24 Schink T, Garbe E. Representativity of dispensations of non-steroidal anti-inflammatory drugs (NSAIDs) in the German Pharmacoepidemiological Research Database. Pharmacoepidemiol Drug Safety 2010;19:\$294.

25 German Federal Agency of Work and Employment [Bundesagentur für Arbeit]. Schlüsselverzeichnis für die Angaben zur Tätigkeit in den Meldungen zur Sozialversicherung 2003. http://www.arbeitsagentur.de/zentraler-Content/ A01-Allgemein-Info/A016-Infomanagement/Publikation/pdf/ SchluesselverzeichnisSozVers.pdf (accessed 11 Apr 2012)

26 Daly LE. Confidence limits made easy: interval estimation using a substitution method. Am J Epidemiol 1998;147:783-90.

27 Kraus N, Chandrasekaran B. Music training for the development of auditory skills. Nat Rev Neuro 2010;11:599-605.

28 Zendel BR, Alain C. Musicians experience less age-related decline in central auditory processing. Psychol Aging 2011;27:410-17.

29 Chesky KS, Dawson WJ, Manchester R. Health promotion in schools of music: initia recommendations for schools of music. Med Prob Perf Art 2006:21:142-4.

30 Langendörfer F, Hodapp V, Kreutz G, et al. Personality and performance anxiety among professional orchestra musicians. J Indiv Diff 2006:27:162-71.

31 Voltmer E, Zander M, Fischer JE, et al. Physical and mental health of different types of orchestra musicians compared to other professions. Med Probl Perform Art 2012;27:9-14.

32 Williamon A, Thompson S. Awareness and incidence of health problems among conservatoire students. Psychology of Music 2006;34:411-30.

33 Altenmüller E, Wiesendanger $\mathrm{M}$, Kesselring J. Music, motor control and the brain. Oxford University Press, 2006.

34 Gan WQ, Davies HW, Demers PA. Exposure to occupational noise and cardiovascular disease in the United States: the National Health and Nutrition Examination Survey 1999-2004. Occup Environ Med 2011;68:183-90. 